easily identified by immunohistochemical profiling techniques. Breast carcinoma subtypes are associated with different clinical outcomes, and targeted therapies might be appropriate in some cases. Here, Siziopikou and Cobleigh suggest that the basal subtype of breast carcinomas might be a candidate for therapies targeted against EGFR.

The authors found that, of 271 consecutive patients with breast cancer, 48 had tumors that expressed the cytokeratin CK5/6-defined as being of the basal subtype. Thirty-one (65\%) of these also expressed EGFR, compared with 39 of $223(17 \%)$ CK5/6-negative tumors $(P<0.0001)$. Thirty-two (67\%) of the CK5/6-positive tumors were ER-negative/PR-negative/HER2-negative; only 28 of 223 (12\%) CK5/6-negative tumors were similarly triple negative $(P<0.0001)$. Of the 48 patients with carcinoma of the basal subtype, 22 were ER-negative/PR-negative/HER2-negative and EGFR-positive.

Many basal subtype tumors are ER-negative/ PR-negative/HER2-negative, and are consequently difficult to treat. The authors propose that such triple-negative tumors should be tested for EGFR expression, and that EGFR-targeted therapies might have benefit in this group of patients who have few other treatment options. A clinical trial of erlotinib-an EGFR inhibitor-in triplenegative EGFR-positive patients is currently in progress at the Rush University Medical Center, Chicago, IL.

Original article Siziopikou KP and Cobleigh M (2007) The basal subtype of breast carcinomas may represent the group of breast tumors that could benefit from EGFR-targeted therapies. Breast 16: 104-107

\section{Cyclo-oxygenase 2 expression correlates with poor prognosis in breast cancer}

There is evidence to indicate that cyclooxygenase 2 (COX2) might promote tumor growth and spread by stimulating angiogenesis and inhibiting apoptosis; however studies examining the association between COX2 expression and prognosis have produced conflicting results. A study by Zerkowski and colleagues has now demonstrated a relationship between high COX2 expression and poor outcome in breast cancer.

The study included 669 stage I-III primary breast cancer specimens analyzed by automated, quantitative analysis (AQUA) of immunostains on tissue microarrays. Low ER levels, large tumor size, positive nodal status and high nuclear grade were significantly associated with poor prognosis in this cohort. COX2 expression was higher in malignant than in benign tissue, and the protein was mainly expressed in the membrane and cytoplasm, with very low nuclear expression. High non-nuclear expression of COX2 was also significantly related to poor disease-specific and overall survival $(P=0.0189$ and $P=0.0055$, respectively). In addition, chi square analysis showed that positive COX2 expression was associated with low ER and PR expression, and with high nuclear grade. In multivariate analysis, high COX2 expression had independent prognostic significance (relative risk [RR] 1.66, 95\% Cl 1.12-2.16), as did both tumor size and nodal status (RR 1.68, 95\% Cl 1.20-2.33 and RR 2.39, $95 \% \mathrm{Cl} 1.67-3.45$, respectively).

The authors conclude that high COX2 expression is an independent prognostic marker for poor survival in primary breast cancer.

Original article Zerkowski MP et al. (2007) Quantitative analysis of breast cancer tissue microarrays shows high cox-2 expression is associated with poor outcome. Cancer Invest 25: 19-26

\section{Good 10-year results with tamoxifen in breast cancer}

A previous report from the first International Breast Cancer Intervention Study (IBIS-I) showed that tamoxifen produced a $32 \%$ reduction in the risk of invasive estrogen receptor (ER)-positive tumors in women who were at increased risk of breast cancer; however, this came at the expense of an increase in the rates of thromboembolic and other events. The results outlined in this report were, however, based mainly on the active treatment period. Now, an updated analysis of the trial demonstrates that the benefits of tamoxifen continue following its discontinuation, while most of the side effects seem to cease.

Women aged $35-70$ years were randomized to treatment with $20 \mathrm{mg} /$ day tamoxifen $(n=3,579)$ or placebo $(n=3,575)$ for 5 years. At a median 96 months after randomization there were 142 breast cancers (invasive plus ductal carcinoma in situ) in the treatment group and 195 breast cancers in the placebo group $(P=0.004)$. The risk of ER-positive invasive breast tumors was $26 \%$ lower in the tamoxifen arm than in the placebo arm during active treatment, but $44 \%$ lower in the subsequent period (up to 10 years). 